\title{
Hardware for digitally controlled scanned probe microscopes
}

\author{
S. M. Clark \\ Division of Biology, California Institute of Technology, Pasadena, California 91125 \\ D. R. Baselt \\ A. A. Noyes Laboratory of Chemical Physics, Division of Chemistry, California Institute of Technology, \\ Pasadena, California 91125 \\ C. F. Spence \\ Division of Biology, California Institute of Technology, Pasadena, California 91125 \\ M. G. Youngquist and J. D. Baldeschwieler \\ A. A. Noyes Laboratory of Chemical Physics, Division of Chemistry, California Institute of Technology, \\ Pasadena, California 91125
}

(Received 18 March 1992; accepted for publication 23 June 1992)

The design and implementation of a flexible and modular digital control and data acquisition system for scanned probe microscopes (SPMs) is presented. The measured performance of the system shows it to be capable of 14-bit data acquisition at a $100-\mathrm{kHz}$ rate and a full 18 -bit output resolution resulting in less than $0.02-\AA$ rms position noise while maintaining a scan range in excess of $1 \mu \mathrm{m}$ in both the $X$ and $Y$ dimensions. This level of performance achieves the goal of making the noise of the microscope control system an insignificant factor for most experiments. The adaptation of the system to various types of SPM experiments is discussed. Advances in audio electronics and digital signal processors have made the construction of such high performance systems possible at low cost.

\section{INTRODUCTION}

Among the most promising applications of scanning probe microcopies (SPM) are those concerned with the study of biomaterials. Although progress has been made in this arena, ${ }^{1-3}$ the routine imaging of biomolecules and biological tissues at nanometer-scale resolution has remained a challenge.

We believe there are at least two barriers to increased resolution during scanning force microscopy (SFM) on biological samples. The first barrier is the limitation on resolution imposed by the shape of the cantilever tip (stylus ). ${ }^{4} \mathrm{~A}$ rounded stylus tends to reduce lateral resolution and to limit tip excursions on highly sloped sample features. The second barrier is the pressure of the stylus on the sample which may degrade image quality by inducing or exacerbating sample motion and deformation. The force exerted on a sample depends not only on the physical forces involved in tip-sample interaction, but is also influenced by the system noise of the SFM. It is only possible to fully exploit tip-sample interactions and other factors which alter microscope resolution if the system noise of the microscope can be made an insignificant factor. The use of sharper styluses to increase resolution may also demand lower system noise to avoid sample distortion and damage, especially when imaging relatively soft samples. We believe that the use of low noise control systems, such as we describe here, is an important step toward realizing the potential of SFM for biology.

The qualities that we feel are most important for scanned probe microscopes to possess are high resolution, low noise, and flexibility. Although several commercial SPMs are available, few are able to achieve the resolution and noise performance that we require for our planned experiments. They can also be difficult to adapt to new approaches (e.g., concurrent optical fluorescence microscopy on biological samples, etc.). We have designed and constructed a high performance digital control and data acquisition system for SPMs in order to increase the resolution of SFM data on biological samples. In this system the output noise may be considered negligible for most experiments and should be adequate for SFM of biomaterials. Due to the modular approach taken, the system is highly flexible and is able to support a wide variety of experiments. With a minimal addition of electronics the system is capable of supporting scanning tunneling microscopy (STM), near field scanning optical microscopy ${ }^{5}$ (NSOM), scanning ion conductance microscopy ${ }^{6}$ (SICM), and scanning electrochemical microscopy ${ }^{7}$ (SECM) experiments. This system may be of interest to others due to its inherent flexibility, low cost, and improved performance.

We will first discuss the ideas behind the use of a digital control system for SPM as well as some of the factors governing ultimate SPM sensitivity and their impact on the design goals for a high-resolution SPM. We then present our instrument as one example of the implementation of a digital control system, concentrating on the design of the analog signal $I / O$ section and performance verification of the circuitry as well as demonstrating the system's flexibility using its adaptation to NSOM as an example.

\section{DIGITAL CONTROL FOR SCANNED PROBE MICROSCOPY}

The minimal set of tasks to be handled by an SPM control system are coarse approach to the sample, the control of a probe micropositioner (usually a piezoelectric ce- 


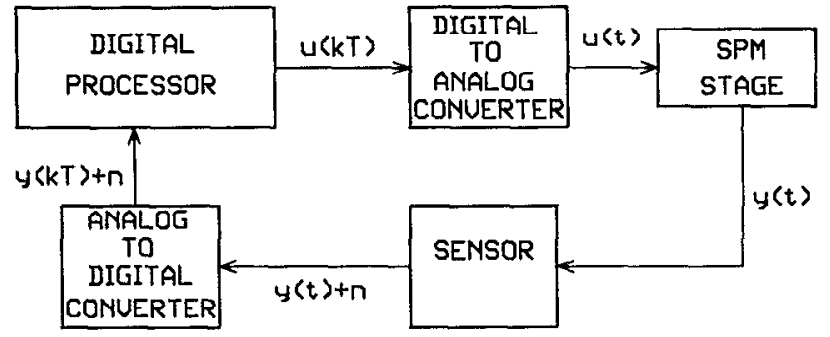

FIG. 1. A block diagram of a digital control system for scanned probe microscopy. Two distinct types of signals occur in a digital control system: (i) those which are time and amplitude continuous (analog signals) denoted $f(t)$; and (ii) those which are quantized in time and amplitude (digital signals) denoted $g(\mathrm{kT})$. In a digital control system both the conversion resolution and the sampling rate effect the degree to which the digital control system approximates an analog control system (Ref. 25). Specialized mathematical techniques which address the digital nature of the signals are used to effectively analyze digital control systems and to insure their performance. In this figure noise is denoted by $n$ regardless of its source.

ramic transducer, hereafter referred to simply as a piezo), and the measurement of a signal which can be used to control the proximity of the probe to the sample. In addition to these elementary functions, the ability to control auxiliary devices and to synchronously acquire multiple types of data is highly desirable.

Discussions of several implementations of control systems for SPMs are available in the literature. ${ }^{8-12}$ Our goals in the design of the present system were to increase performance by avoiding some of the compromises which had been made in other systems and increase system flexibility through a modular design approach using a digital signal processor (DSP). Despite the availability of DSPs in commercial SPMs, we are unaware of any detailed discussion of DSP based control systems as applied to SPMs. Hence, we will review some of the principles involved below.

In a digital control system for SPM (see Fig. 1) the proximity signal (tunneling current in STM, cantilever deflection in SFM, etc.) is measured by a sensor using analog signal processing electronics and digitized by an analog to digital converter (ADC). The digital value representing the signal is compared against a digital setpoint to obtain an error signal. This error signal is used to calculate a digital value representing the ideal voltage to be applied to the $Z$ axis of the micropositioner. The algorithm used for this calculation comprises the $Z$ feedback loop. This new $Z$ value is converted into the actual $Z$ voltage by a digital to analog converter (DAC) and amplified by a high voltage amplifier to provide the output voltage necessary to drive the micropositioner. Although it is possible to correct for nonlinearities inherent in the piezoelectric actuators through position feedback, ${ }^{13}$ typically the voltages used to drive the micropositioner in the $X$ and $Y$ dimensions are generated in an open loop fashion (that is, they are calculated and sent to the $X$ and $Y$ DACs without measuring a control signal for them).

The major advantages offered by a DSP-based control system are flexibility, improved performance (decreased scan times, increased noise immunity, etc.), and the possi- bility of easily using sophisticated techniques such as phase sensitive detection, optimal filtering, active damping, evolutionary feedback parameter optimization, and so forth. The tremendous flexibility of a DSP-based system arises from the generic nature of the analog $I / O$ electronics combined with the ability to modify the control software. This allows the implementation of novel imaging modes or the adaptation of the system to control different devices with a minimum of hardware development thus saving both time and expense. Another advantage lies in the capabilities of the DSP itself. Because DSPs typically contain features such as single machine cycle multiplication and matrix manipulation facilities, they are particularly well suited to real-time computation and signal processing. They are much faster for these tasks than microcontrollers or other dedicated microprocessors. The last barrier to the use of DSPs for SPM control systems was removed with the advent of low cost, high performance DACs and ADCs for application in the audio frequency range.

Another key to system fiexibility is to use a modular design approach. By breaking the system into generic components which can be controlled through a standard interface it is possible not only to simplify component design, but also to update easily the system configuration as requirements change. For more elaborate experiments, modules can simply be added which follow the standard communication protocol and which perform specialized functions.

As an illustration of the potential engendered by modular design, we show, in Fig. 2, a block diagram of a multiprocessor architecture. This type of architecture allows task sharing between the central DSP and other embedded processors. In this system, "smart" DACs could serve to relieve the DSP of raster generation and other peripheral control tasks, these tasks being handled by embedded microprocessors (either DSPs or microcontrollers). Through the use of such "smart" DACs the system could be tailored to particular piezos and real time hysteresis and creep correction could be done. One way in which this might be achieved is by implementing the real time optical scan correction system of Barrett and Quate ${ }^{13}$ using a digital servo loop. In such a design the optical beam displacement sensors would provide a measurement of the piezo displacement and the desired $X-Y$ raster pattern becomes a twodimensional, time-dependent reference input to the servo. The implementation of various types of control loops such as proportional-integral-differential (PID) control, optimal control, or feedforward control could be easily done in the control software. A particularly attractive microcontroller for this application might be the Motorola MC68332 14 due to its hardware interpolation feature which would allow rapid generation of smooth raster signals. The field programmable gate array (FPGA) shown in the diagram allows complex timing and logic functions to be handled easily and facilitates the interface between the processors. The "smart" module concept can be extended to more interesting applications such as microprocessor controlled scanning bipotentiostats for scanning electrochemical microscopy and so forth. 


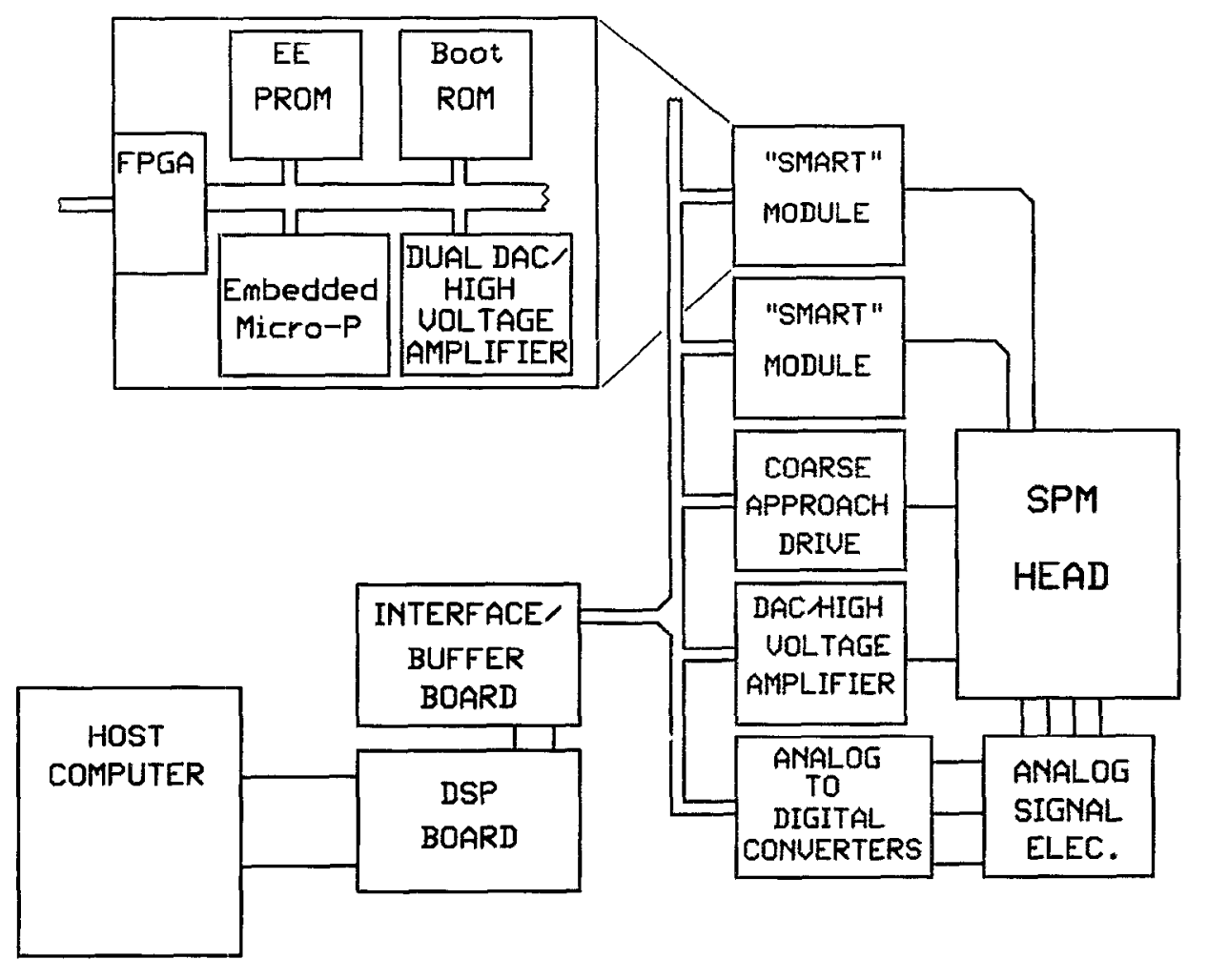

FIG. 2. A block diagram of a multiprosessor control and data acquisition system for scanned probe microscopy. This architecture allows task sharing between the central DSP and other embedded processors. In one version of such a system the central DSP would be used to execute the $Z$ feedback loop and store image data, while the other processors could be used for raster generation, digital rotation, control of auxiliary equipment (e.g., a scanning bipotentiostat, hybrid photomultiplier detectors, etc.).

\section{NOISE IN SPM}

The noise level of a SPM control system is a significant factor in its ability to meet experimental demands. To understand how noise influences SPM experiments, and to provide a framework for minimizing noise, one can divide noise sources into two categories, output noise and input noise. Output noise originates in the piezo drive electronics and can be minimized by the use of special low noise design techniques. Input noise is inherent in SPM measurements and cannot be reduced beyond a certain minimal level. We discuss both noise categories below.

Reduction of piezo drive output noise was one of our major design goals. Because the force applied by a cantilever is proportional to its deflection, noise on the $Z$ axis output lying outside the servo bandwidth introduces uncontrollable force fluctuations which are undesirable (within the servo bandwidth the noise will be reduced by the feedback gain). Our specific design goal for the SFM was that the system noise contribution from the piezo drive electronics be slightly less than the mechanical noise contribution from the microscope stage and head; from calculation we expect the mechanical noise from thermal stage vibration to be $\approx 0.1-\AA$ rms amplitude. We frequently use tubular piezos and a response of $\approx 50 \AA / \mathrm{V}$, hence our system can tolerate an output noise of $2-\mathrm{mV}$ rms over a $100-\mathrm{kHz}$ bandwidth while maintaining a $1-\mu \mathrm{m}$ scan range.

Resolution is another key consideration; ideally the cantilever could be positioned at any arbitrary deflection.
The average incremental force applied by the stylus depends on the DAC output step size, with smaller steps providing finer control of the applied force. Typically, higher resolution DACs also exhibit lower output noise, so high-resolution DAC circuitry provides a real advantage in terms of controlling forces for SPMs. Large zoom ratios are another feature gained by using high resolution DACs. The ability to zoom in on rare features initially found by scanning large arcas can be crucial in systcms without sample translators and greatly simplifies the use of any SPM.

In almost every experimental measurement, weak input signal detectability is limited by noise. In many cases interfering signals generated externally to the experiment may be excluded from the measurement by proper shielding and grounding, however, noise can be reduced only to a finite limit. This ultimate noise floor is the result of the thermal motion of charge carriers (Johnson noise) and the quantization of charge (shot noise). The voltage noise (rms) due to Johnson noise of a resistance $R$ is given by ${ }^{15}$

$$
V_{n}=(4 k T R B)^{1 / 2}
$$

In this equation, $T$ is the absolute temperature $(\mathrm{K}), B$ is the measurement bandwidth $(\mathrm{Hz})$, and $k$ is Boltzmann's constant $\left(1.380662 \times 10^{-23} \mathrm{~J} \mathrm{~K}^{-1}\right)$.

Just as Johnson noise from a source resistance presents a fundamental limit for voltage measurements, shot noise presents a fundamental limit in the experimental measure- 
ment of currents. The shot noise (rms) for a current of average value $\langle I\rangle$ is ${ }^{15}$

$$
i_{n}=\{2 q\langle I\rangle B\}^{1 / 2},
$$

where again, $B$ is the bandwidth of the measurement $(\mathrm{Hz})$ and $q$ is the electron charge $\left(1.602189 \times 10^{-19} \mathrm{C}\right)$. The best measurements one can make are limited by noise at these levels. Only Johnson noise can be decreased by lowering the temperature of an experiment; however, both shot noise and Johnson noise can be reduced by limiting the detection bandwidth. By using techniques such as lockin detection, bandwidths of a few Hertz can be readily obtained.

When one considers the effect of shot noise on STM experiments one finds that for a tunneling current of 100 $\mathrm{pA}$ and a $10-\mathrm{kHz}$ bandwidth the shot noise is $0.4 \mathrm{pA}$. Thus, the maximum signal-to-noise ratio for these conditions is $\approx 250$. Similarly, for a $1-\mathrm{pA}$ tunneling current, in the same $10-\mathrm{kHz}$ bandwidth, the best signal-to-noise ratio is less than 20. Often the effects of shot noise are not obvious in topography data from STM; this is due to the fact that high impedance amplifiers typically used as STM preamplifiers tend to have a limited frequency response, effectively limiting the bandwidth to $\approx 1 \mathrm{kHz}$ or less. One might expect, however, that shot noise would play a more significant role in experiments done at high gap resistances, over large bandwidths, or in tunneling spectroscopy.

Shot noise also limits optical beam deflection sensitivity for SFM. For a photodiode, $\langle I\rangle$ is given by $\langle I\rangle=P \varnothing$, where $P$ is the optical power incident on the photodiode and $\varnothing$ is the spectral responsivity at the wavelength of interest. Under typical operating conditions in our SFM, $\langle I\rangle$ is $\approx 15 \mu \mathrm{A} /$ quadrant and $i_{n}$ is $\approx 690 \mathrm{pA} /$ quadrant assuming a $100 \mathrm{kHz}$ measurement bandwidth. Thus, in our SFM as presently used, current fluctuations of amplitude $2 i_{n}$ give rise to signals of $\approx 280 \mu \mathrm{V}$ which roughly corresponds to a change of one ADC unit ( $\pm 10 \mathrm{~V}$ full scale and 16-bit resolution). In the sense that our instrument can detect changes in photocurrent of amplitude $2 i_{n}$, we can say that it is shot noise limited. Further increasing the gain of the difference amplifier, whose output is the deflection signal, provides larger signal amplitude, but does not increase signal-to-noise ratio.

To increase sensitivity further requires increasing the laser power on the photodiode, increasing the optical lever gain (i.e., the ratio of beam deflection distance to cantilever tip motion) or decreasing the measurement bandwidth. The laser power can easily be increased; however, as the light intensity on the cantilever increases so does the momentum transferred to the cantilever. The force exerted on the cantilever by photon pressure is given by

$$
F=2 P / c \text {, }
$$

where $P$ is the laser power incident on the cantilever and $c$ is the speed of light. Thus, for a helium-neon laser with $10 \%$ intensity noise, to keep laser noise induced force fluctuations below $10^{-11} \mathrm{~N}$ one is limited to less than $\approx 20$ $\mathrm{mW}$. On the other hand, the optical lever gain can be incrcased without compromising other aspects of the instrument's performance. However, optical lever gain is limited in practice by the mechanical design of the instrument both in terms of mechanical noise and ease of use.

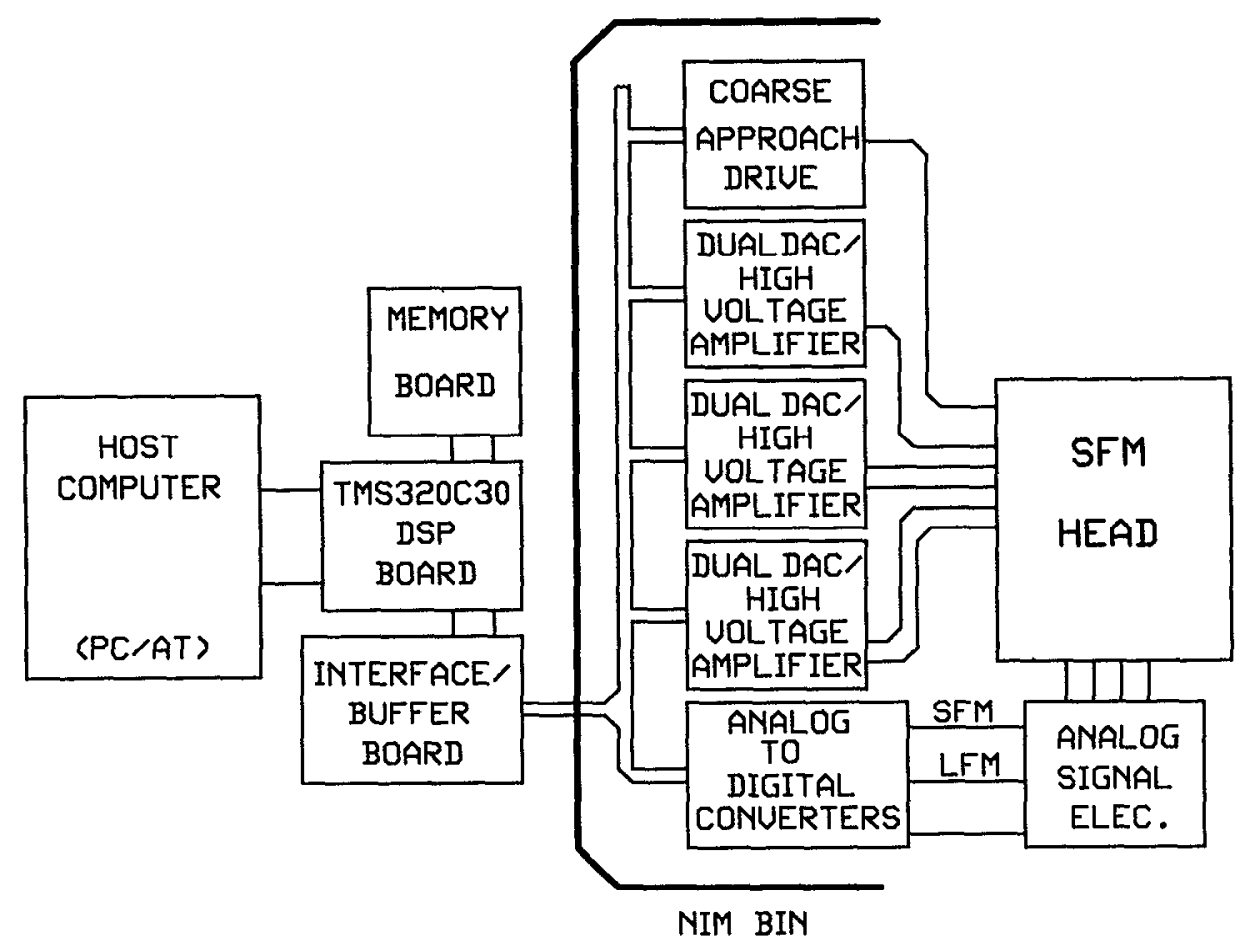

FIG. 3. A block diagram of the scanned probe microscope control and data acquisition system. The NIM (nuclear instrumentation module) bin is indicated only schematically and all power supplies have been omitted for diagram clarity. Other host systems and alternative packaging could clearly be used (e.g., workstations and VXI bus). 
To interpret the shot noise limit in terms of position sensitivity for an optical lever based SFM requires knowledge of the diameter of the reflected beam at the photodiode face and the optical lever gain. For the SFM head presently used, the beam diarneter at the photodiode is $\approx 10 \mathrm{~mm}$ and the optical lever gain is $\approx 2500$ when using a $100-\mu \mathrm{m}$ cantilever. Although the beam on the face of the photodiode has a Gaussian intensity profile, a worst case shot noise sensitivity value may be calculated by assuming a uniform intensity distribution. Using this approximation, with a $20-\mathrm{mW}$ incident on the photodiode the shot noise limit corresponds to a position sensitivity of $\approx 0.05 \AA$.

\section{SYSTEM OVERVIEW}

The system which we have designed and are presently using for experiments is based on a single DSP. We opted to purchase a board level DSP and to focus effort on developing the algorithms and software for SPM control as well as the analog $I / O$ described in this paper. As can be seen in Fig. 3, the major components of the digital control and data acquisition system are the DSP, the piezo drivers, the analog signal processing section, and the ADCs. Because the analog signal processing electronics are instrument specific they will not be discussed in detail here.

For our system we chose the Spectrum TMS320C30 DSP board ${ }^{16}$ which uses a personal computer (PC) as the host. The PC also serves for software development and image processing and display tasks. Due to the noise level introduced onto the DSP board by the PC, we found the on-board analog $I / O$ to be inadequate for our needs, hence our custom designed analog $I / O$ uses the external interface bus (DSP-Link).

For our laboratory the most cost effective method of packaging the analog $I / O$ was to modify nuclear instrumentation module (NIM) bins by adding a digital backplane and to use commercially available NIM enclosures to house individual printed circuit boards. Using the NIM format forces design modularity and facilitates the exchange of modules between microscopes. We have buffered the DSP-Link signals and used these to drive directly the terminated digital backplane. This implementation of a generic high-speed digital bus allows zero wait state communication between the NIM modules and the DSP; in fact, by using high-speed bus drivers the present hardware could easily accommodate bus bandwidths in excess of 50 Mbytes/s. Other NIM bin modifications include the addition of an independent $+5-\mathrm{V}$ linear power supply for powering the digital circuitry and the addition of high voltage power supplies.

\section{SYSTEM COMPONENTS}

\section{A. Digital signal processors}

For most users the selection of a DSP for application in a SPM system will entail the selection of a board level component for a particular computational platform (personal computer, workstation, etc.). The major issues involved in the selection of a DSP board, such as floating point or integer operation, memory addressing capability, and the quality of on-board $I / O$ functions are discussed in Ref. 17. Although the electronics described in this paper will function with any DSP board having an external bus, processor speed is an important factor if fast scan rates are required. Processors that have fast clock rates allow either more operations to be performed in the feedback loop or faster sampling intervals. At the time this work was begun, a TMS320C30 DSP(Texas Instruments ${ }^{18}$ ) based board from Spectrum Signal Processing was chosen due to its ready availability and reasonable price. This board has a $33-\mathrm{MHz}$ DSP clock rate which allows 150 instructions to be executed in the interrupt interval used in our system (10 $\mu \mathrm{s})$. We find that the $100-\mathrm{kHz}$ sampling rate easily permits a feedback servo bandwidth of $20 \mathrm{kHz}$ and that $150 \mathrm{in}$ structions are adequate for the implementation of the $Z$ feedback loop and the scan raster generation. Since the time of our purchase, board level products suitable for SPM control based on several different DSPs have come onto the market providing a variety of sophisticated products to choose from.

If the capabilities of board level DSP products prove to be inadequate for a particular microscope design, one may be forced to design a DSP board from scratch. This is obviously not a trivial undertaking and should only be done as a last resort; however, some points merit consideration.

The first point is that the performance of the $Z$ feedback loop and its complexity are constrained by the speed of the DSP. Second, because the feedback loop is a realtime process, only minimal time delay can be tolerated without compromising scan rate. There are two potential areas in which to gain performance: (i) DSP speed, (ii) relieving the DSP of tasks easily handled by less sophisticated processors. Other advantages gained by the design of a SPM specific DSP board are the ability to place substantial amounts of fast memory and/or dual port memory on the board as well as the ability to use the serial data ports and external buses of the DSP to interface directly with the DACs and ADCs.

\section{B. Piezoelectric ceramic drive electronics}

Because we require high-resolution digital to analog converter (DAC) outputs we considered two ways to achieve high resolution: (i) by using a single highresolution DAC; and (ii) by scaling the outputs of two or more lower resolution DACs and summing their outputs. Previously one would have been forced to implement the latter alternative, however, recently, tremendous progress has been made in the performance of low cost audio frequency DACs. With the availability of these components, the single DAC alternative becomes more attractive due to its simplicity.

Several manufacturers produce DACs which offer greater than 16-bit resolution with total harmonic distortion plus noise of less than $-96 \mathrm{~dB}$. Typical of this class of DAC is the PCM58P-K from Burr-Brown ${ }^{19}$ which we have used in our SPM system. This is an 18-bit serialinput, current-output DAC with an idle channel signal to noise ratio of $+126 \mathrm{~dB}$ at bipolar zero. This DAC per- 


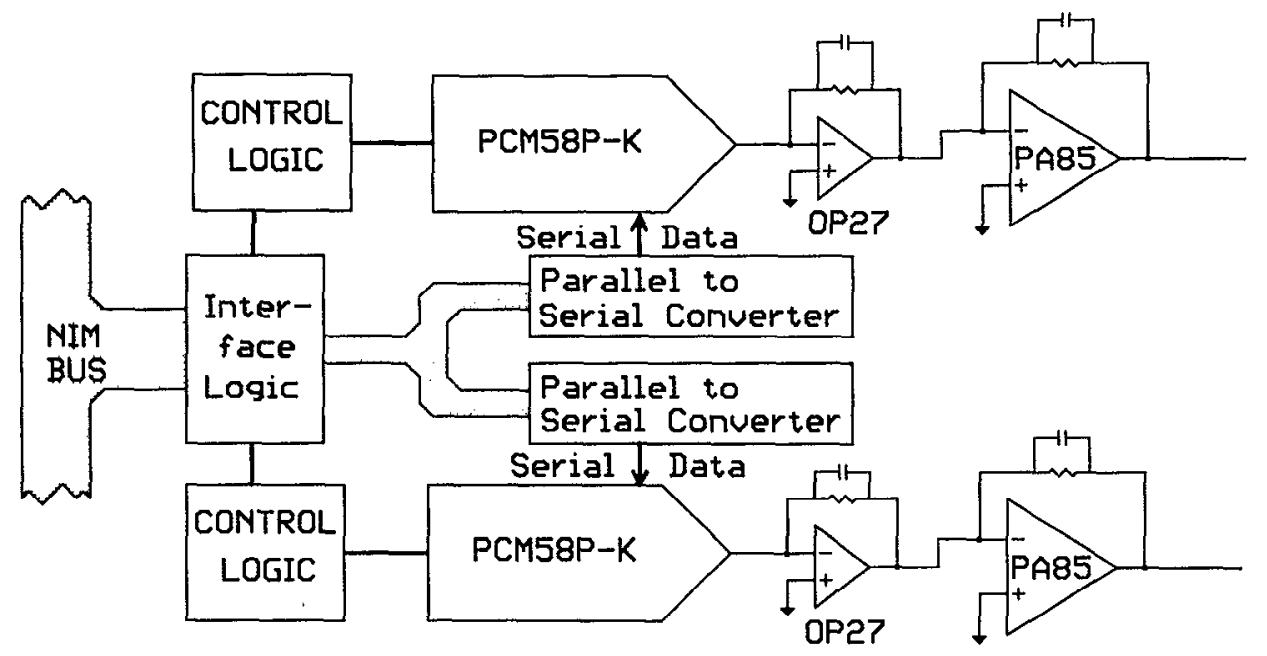

FIG. 4. A diagram of the piezoelectric ceramic electronics. The piezodrive electronics are composed of dual DACs and high voltage amplifiers. The interface logic consists of data and address buffers and address decoders. The address decoders are implemented in programmable array logic (PAL) devices. The control logic is also implemented in PALs and functions to provide the required clock and control signals for the DACs and the parallel to serial converters (shift registers).

forms suitably in SPM applications in our laboratory; however, even higher performance DACs such as the PCM63P (Burr-Brown) have recently come on the market. The PCM63P offers 20-bit resolution with low noise and for new designs this type of DAC should also be considered.

For our system the selection of high voltage amplifiers to drive the piezos was uncomplicated due to the narrow field of parts to choose from. Component level high voltage operational amplifiers suitable for SPM applications are available from primarily two companies, Apex Microtechnology ${ }^{20}$ and Burr-Brown. The major considerations in judging a high voltage amplifier for use with piezoceramic scanners in SPM are (i) total output voltage swing, (ii) output noise, and (iii) current drive capacity. The current capability required of an amplifier is an important consideration because of the capacitance presented by the piezoceramic to the output of the amplifier. The output currents required of the amplifier to achieve a given output voltage slew rate may be found from $i=C d V / d t$. We used the PA85 from Apex because at the time it offered the greatest output voltage range at a suitable current capability.

\section{Piezoelectric ceramic drive performance}

A schematic diagram of a piezodrive module is shown in Fig. 4. The piezodrive electronics are composed of dual DACs and high voltage amplifiers. The interface logic consists of data and address buffers and address decoders. The address decoders are implemented in programmable array logic (PAL) devices. The control logic is also implemented in PALs and functions to provide the required clock and control signals for the DACs and the parallel to serial converters (shift registers).

The assembly is physically composed of two printed circuit boards mounted in a double width NIM module; one board contains the dual DAC circuitry and the other board contains the high voltage amplifiers. This division of the circuitry allows the use of the DAC section alone for low voltage applications (e.g., tip-sample bias generation) without having to fabricate special boards.

A theoretical analysis of the DAC/HV amplifier circuitry would predict that with a $420-\mathrm{V}$ output range, the output noise of this section should be $\approx 100-\mu \mathrm{V}$ rms in a $100-\mathrm{kHz}$ bandwidth (discounting $1 / f$ noise below about 10 $\mathrm{Hz}$ ). Figures 5 and 6 present data to document the time

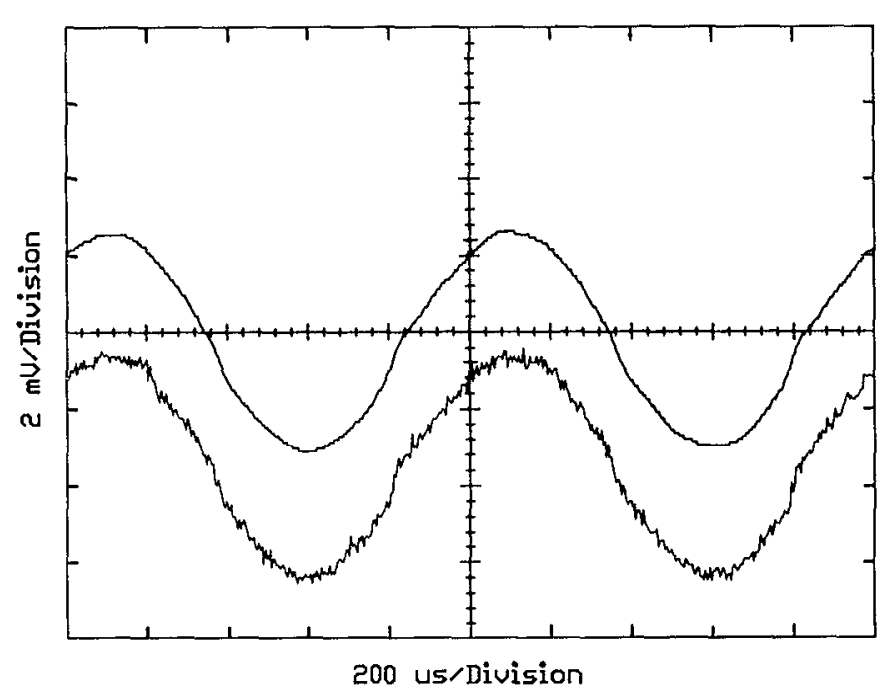

FIG. 5. Measured time domain characteristics of the $420-\mathrm{V}$ range piezodrive electronics. The upper trace presents the average of 256 time traces taken by a digital storage oscilloscope (Tektronix 2440). The waveform amplitude is $\approx 5 \mathrm{mV}(-98 \mathrm{~dB})$ and its frequency is $\approx 1 \mathrm{kHz}$. This representation eliminates random noise (as it averages to zero) while maintaining any systematic features of the signal. The structure seen in the waveform is due to oscilloscope digitization and not individual DAC steps. The lower trace presents a single time trace of the same amplitude and frequency so that noise contribution may be seen. At this signal level the output consists of only three DAC steps but they are not visible due to the output low pass filters. 

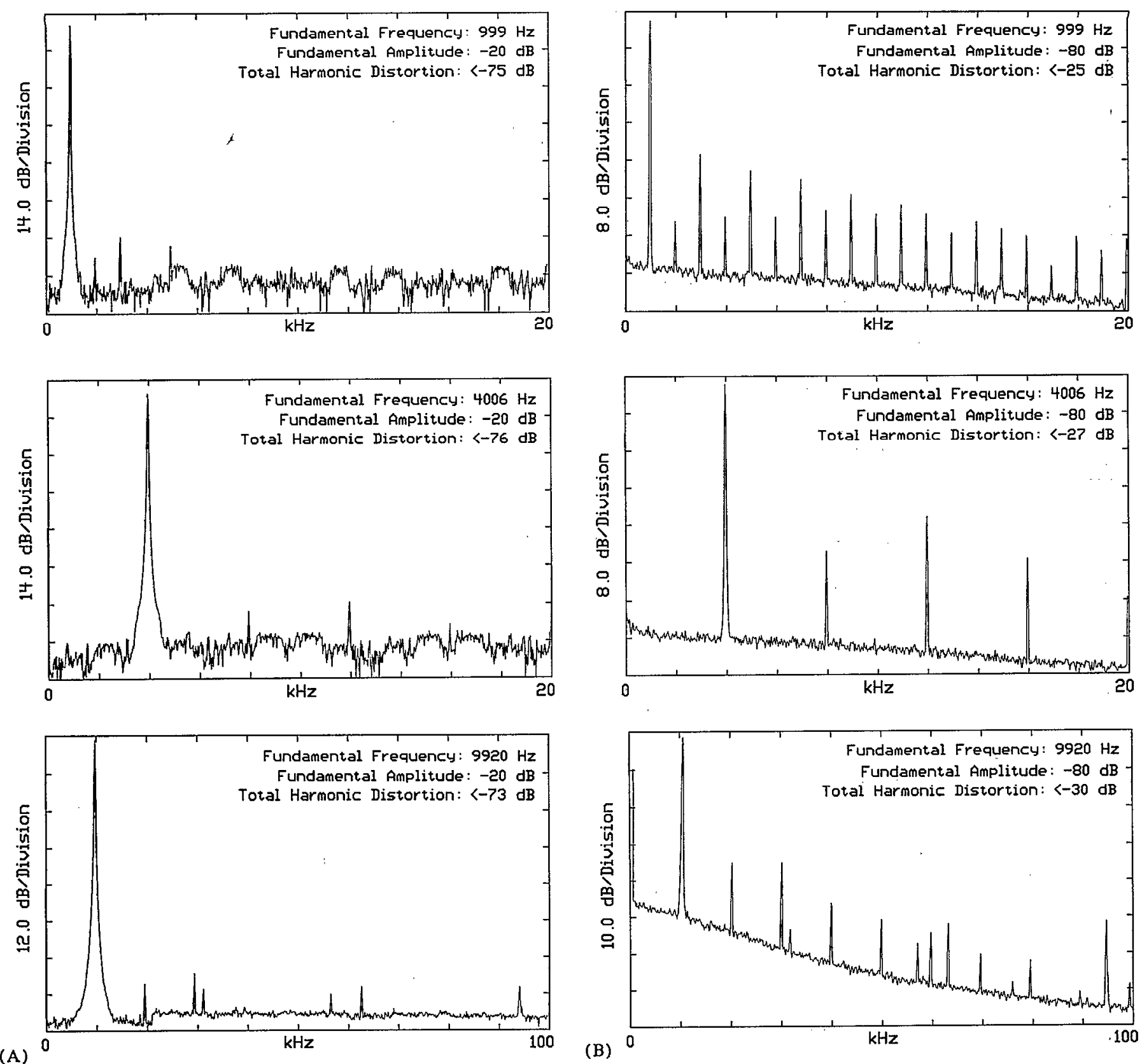

FIG. 6. Measured frequency domain characteristics of the 420-V range piezo drive electronics. Power spectra of the piezo drive electronics taken to assess the total harmonic distortion at various frequencies and signal amplitudes. The spectra were taken using a Hewlett-Packard 3563A Control Systems Analyzer. A resistor divider was used to scale the signal to avoid overloading the analyzer input circuitry. For all of the spectra, the analyzer was set to 50 time record averages. Note that the total dynamic range (maximum output signal/noise level of the output) of the piezo drive is $\approx 118 \mathrm{~dB}$. (A) -20 -dB signal level spectra. The total harmonic distortion is $<-75 \mathrm{~dB}$. (B) $-80-\mathrm{dB}$ signal level spectra. The total harmonic distortion is $<-25 \mathrm{~dB}$.

domain and frequency domain performance of the DAC/ HV amplifier section. As can be seen in the time domain traces of Fig. 5, the broadband output noise of this section (without feedback) is $450-\mu \mathrm{V}$ peak to peak $(320-\mu \mathrm{V} \mathrm{rms,}$

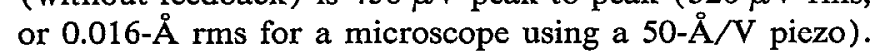
This gives a measured dynamic range (maximum output signal/noise level of the output) of $\approx 118 \mathrm{~dB}$.

In Fig. 6 we show power spectra measured to judge if the harmonic distortion of the DSP/HV amplifier section would present a problem. To perform this test of the circuitry, digital representations of the sine waves of various amplitudes and frequencies were generated and output via the piezo drive circuitry. The power spectra were measured using a Hewlett-Packard 3563A Control Systems Analyzer. An ideal spectrum would have a single peak (strictly speaking, a delta function) located at the frequency of the sinc wave with no other features. Two aspects of these spectra are of particular interest; the broadband noise can again be seen to be negligible (the baseline of the spectra match the recording instrument noise floor) and the spectral purity of the output signals eliminates concerns that nonlinearity in the drive electronics might give rise to noise in the $Z$ feedback loop or excite undesired resonances in the piezotube. 
When examined together, the data of Figs. 5 and 6 document a level of output performance which compares very favorably with completely analog SPM control systems and was previously unattainable with low cost digital systems. The outstanding performance of this circuitry strongly suggests that this section should function adequately for most SPM applications. In the event that this level of performance is inadequate, further reductions in the output noise level and signal purity may be obtained by using better high voltage power supplies in conjunction with better electrical shielding.

\section{Analog to digital converters}

In the area of analog to digital converters (ADCs), again striking progress has been made both in cost and performance. Recently several companies have introduced parts which offer 16 bit and greater resolution. Two architectures dominate the field: (i) the delta/sigma type and (ii) the successive approximation type. ${ }^{21}$ We have found that the conversion time, defined as the time interval between the sampling of a signal at the start of the conversion process and the end of the conversion at which time the digital value is available for the servo loop to act on, typical of commercially available delta/sigma converters (on the order of $1 \mathrm{~ms}$ ) would severely limit feedback loop bandwidth. Thus we have chosen the PCM78P from BurrBrown, a 16-bit successive approximation converter which offers a $5-\mu$ s conversion time with better than $-80-\mathrm{dB}$ signal-to-noise ratio. Although the PCM78P claims 16-bit resolution, it is specified as having only 14-bit linearity and no missing codes at 14-bit resolution.

The other components which determine the performance of the ADC section are the anti-aliasing filter and the track and hold amplifier. The practical criterion which must be met by the anti-aliasing filter is that signals in the frequency region above half the ADC sampling frequency must be attenuated to less than one least significant bit. We have found the APQ-25 series of passive filters from Toko America $^{22}$ to be more than suitable in this role. The particular part used in our system is a seven pole low pass filter with a constant $\approx 35-\mu$ s group delay (the time taken for a signal to pass through the filter) throughout the passband and a nominal cutoff frequency of $\approx 30 \mathrm{kHz}$. Passive filters are preferred for low noise applications because passive components add a minimum of noise to the processed signal.

The track and hold amplifier used in conjunction with the ADC must be compatible with the desired system performance (i.e., a 12-bit track and hold will degrade the performance of a 16-bit ADC). As specified by Analog Devices, ${ }^{23}$ the AD1154BW track and hold amplifier is compatible with our design goals. The AD1154BW is a true 16 bit, low cost track and hold amplifier with a $2-\mu \mathrm{s}$ settling time. It performs well in our SPM.

\section{E. ADC section performance}

Figure 7 presents a schematic of a single ADC section. The four ADC sections contained on a single ADC board

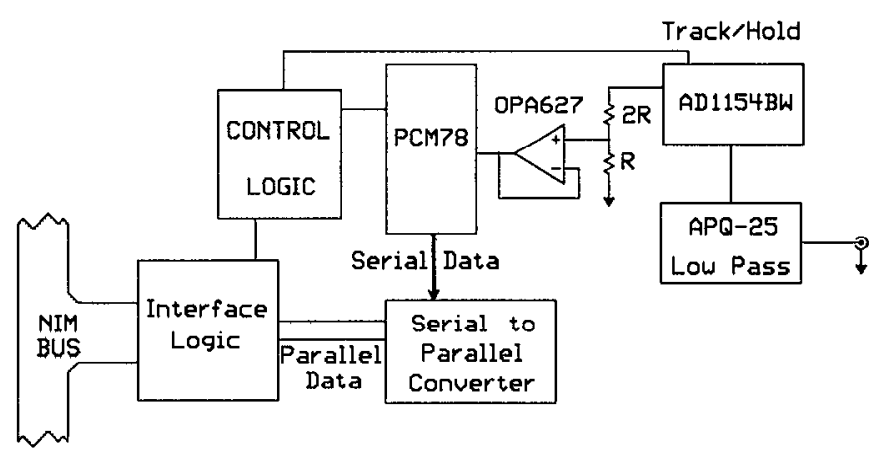

FIG. 7. A diagram of a single-channel analog to digital converter for the scanned probe microscope control and data acquisition system. The resistor divider is included to correctly scale the input to the ADC and the operational amplifier (OPA627) serves to buffer the divider voltage from the dynamic $1.5-\mathrm{k} \Omega$ input impedance of the ADC. In the prototype system there are four such channels on the ADC board. The interface logic consists of data and address buffers and address decoders. The address decoders are implemented in programmable array logic (PAL) devices. The control logic is also implemented in PALs and functions to provide the required clock and control signals for the ADCs and the serial to parallel converters (shift registers).

are housed in a double width NIM module. One convert command triggers the conversion start for all of the ADCs, thus avoiding any question concerning the phase relations of the individual ADCs. For SFM we use three of the ADCs; one for vertical cantilever deflection, one for torsional cantilever motion, and one for total light intensity on the quadrant photodiode. An alternative scheme for SFM is to directly digitize all four photodiode quadrant signals. This approach has the advantage that an almost perfect common mode signal rejection ratio can be obtained. In order to do the digital signal processing required by digitizing all of the quadrant signals the sampling interval would have to be lengthened and this might limit the $Z$ feedback servo bandwidth. In our system we might have to increase the sampling interval from 10 to $15 \mu$ s to provide the required computational time.

The design goal for the ADC section was to achieve full 16-bit measurement capability with a $\pm 10-\mathrm{V}$ input range at a sampling rate in excess of $100 \mathrm{kHz}$. This would give four times oversampling of the highest frequency signals of interest, thus meeting the requirements of the $Z$ feedback loop and allowing high scan rates. The ADC section achieves a maximum conversion time of $\approx 6 \mu \mathrm{s}$ allowing a sampling rate in excess of $150 \mathrm{kHz}$. At this sampling rate the ADC section yields 14 bit performance which is limited by ADC noise of 2 bits ( 4 ADC units or $1.2 \mathrm{mV}$ referred to the $A D C$ section input).

We have done a more detailed evaluation of the ADC circuitry for missing codes (at 12-16 bit resolution levels) and differential linearity ${ }^{21}$ which is shown in Fig. 8. To do the evaluation we use a low-frequency linear bipolar ramp as the input waveform to the ADC. By using this waveform and acquiring data over many cycles of the ramp we should accumulate a significant number of samples at each of the ADC output codes. We then construct a histogram which has each of the output codes along the abscissa and the number of samples at each output code as the ordinate. 

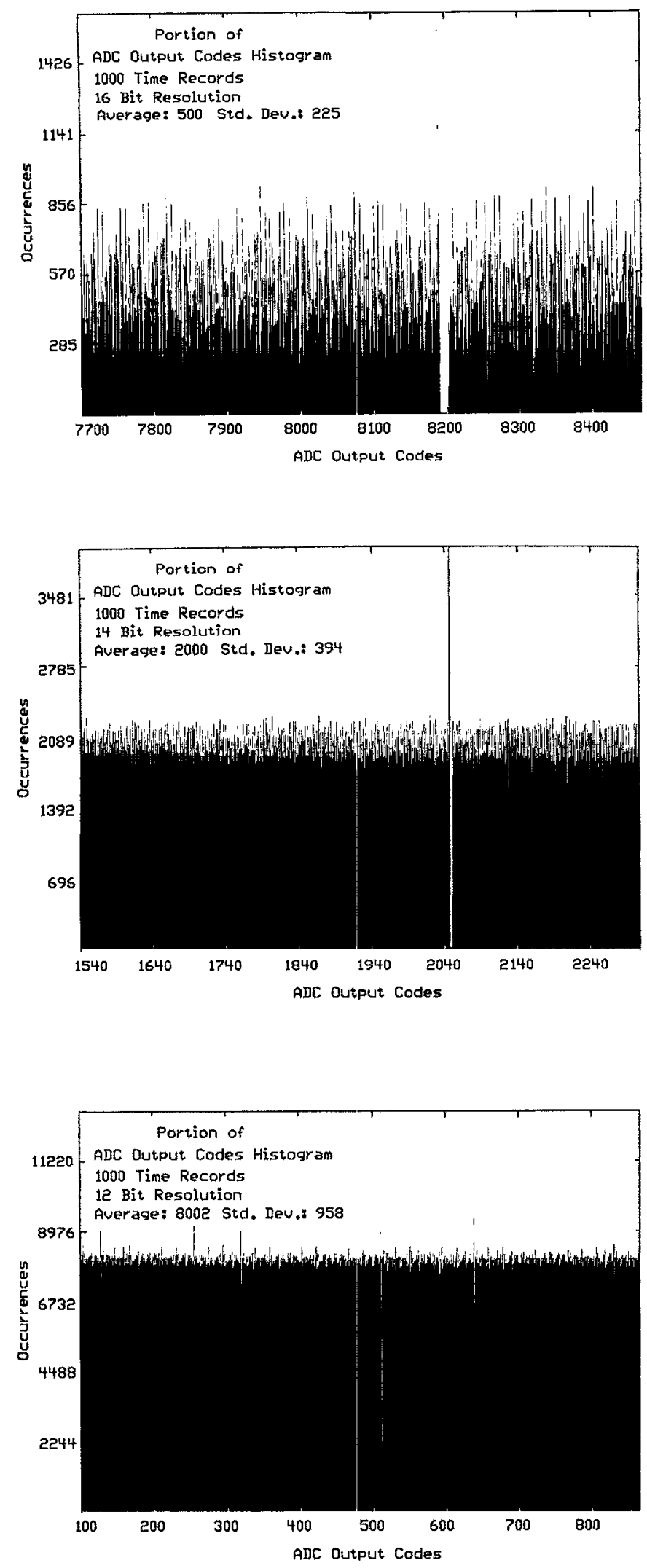

FIG. 8. Measured performance of the analog to digital converter section. A typical section of ADC output code histogram at three resolution levels. The same area of missing codes is presented in each of the histograms at a different resolution level so that the effects of rebinning would be clear. The sections of histograms shown were selected because they are illustrative of clusters of missing codes found in the ADC output. All of the histograms are derived from a data set consisting of 1000 time records.
For an ideal ADC this histogram would have an average number of samples in each of the bins with the expected deviation from this being given by the square root of the number of samples expected in the bin. If the ADC has missing codes one would expect that there would be no samples falling within the bin for the missing code. Differential linearity may also be coarsely judged by this technique; bins which show deviation from the expected value of samples in the bin by more than the standard deviation can be judged to have a linearity problem. In Fig. 8, two aspects of the histogram structure are noteworthy. First, we find that there are indeed bins with no samples and that if we reduce the effective ADC resolution by rebinning (i.e., to bin at 15 -bit resolution we combine adjacent bins from the 16-bit histogram, etc.) it is only when we reach the 12-bit resolution level that there are no missing codes. Second, the histograms show significant deviations from ideal behavior in both periodic structure and deviation from the value expected in the bins.

One can view the number of counts in a bin as representative of the width of a bin (i.e., the voltage range resulting in a count being added to a particular bin). Adopting this view, one finds from Fig. 8, that bins corresponding to missing codes often have very wide bins adjacent to them. Thus, if one regards the ADC output code histogram as constituting a type of transfer function for the ADC, one could increase ADC performance by post-processing data to normalize bin width (reduce differential linearity error to zero).

In order to summarize the differential linearity measurement we have constructed a second type of histogram, shown in Fig. 9, called the count distribution histogram. In this histogram we display the number of counts in a bin (bin value) as the abscissa and the number of bins which contain that bin value as the ordinate. Again, if the ADC performance were ideal, this should produce a distribution with a single mode located at the average number of samples in a bin. The area of the histogram equals the total number of samples and the standard deviation of the distribution would be found from Poisson statistics to be the square root of the average number of samples expected in a bin. As can be seen in Fig. 9, the stringency of this test technique varies with the number of samples. In the small sample cases, 10 and 100 time records, the distribution does not show significant departure from ideal behavior (i.e., the distributions show one mode and Poisson character). However, in the large sample cases there is a clear deviation from ideal. The appearance of multiple maxima in the distribution indicates that ADC bin widths are not uniform, but rather, their widths occur in clusters. The clustering of bin widths at values distinct from the mean necessarily implies differential nonlinearity. Although these test data indicate that the differential linearity of PCM78P leaves a great deal to be desired, we find in practice, that the signal-to-noise ratios of our SPM system are sufficiently large that missing codes and nonlinearities in the ADC circuitry do not seriously degrade instrument performance (e.g., four consecutive ADC codes for $\pm 10-\mathrm{V}$ input range at 16-bit resolution corresponds to a "dead 

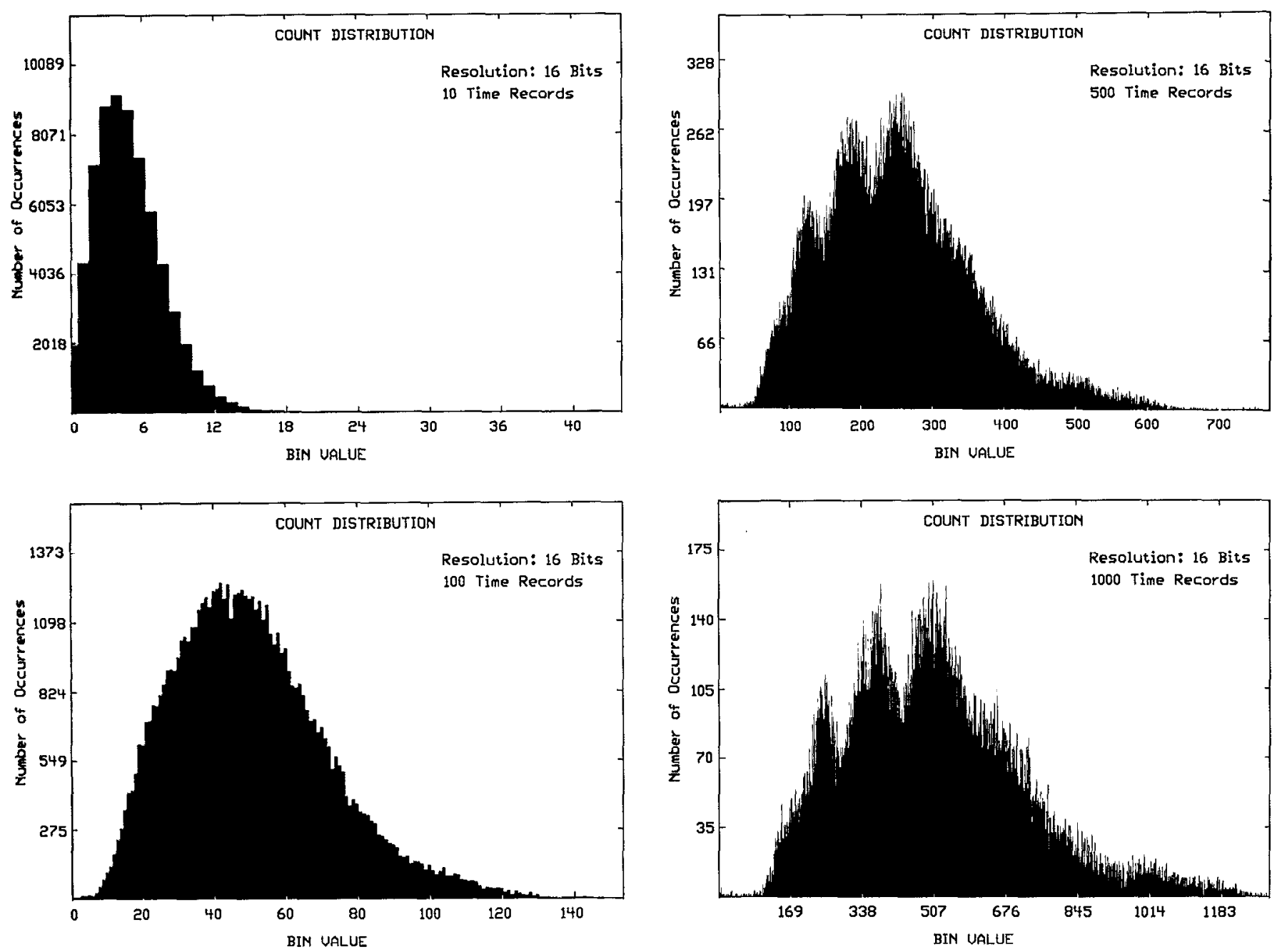

FIG. 9. Evolution of the count distribution histogram with sample size. These histograms demonstrate the general Poisson character of the count distribution histogram. The construction of the histograms from the binned data of Fig. 9 is described in the text. In the histograms with larger numbers of samples one can see not only significant deviation from an ideal Poisson distribution, but also the multimodal character of the histogram. These data are indicative of the poor differential linearity of the PCM78P.

band" of $\approx 1.2 \mathrm{mV}$ ). For systems in which this is not the case, we believe that the diagnostic tests outline above may prove useful in the selection of ADCs.

Although we are not able to achieve 16-bit performance, we would point out that a 14-bit performance level is tolerable for most SPMs, and that systems which offer performance superior to ours are much more expensive (by factors of 5-10). Also, as mentioned previously, the adaptation of the system to accommodate higher performance $\mathrm{ADCs}$ as they are required is quite straightforward. One should also note that, for some SPM designs, high-speed true 12-bit ADCs may be an optimal ADC choice.

\section{SYSTEM PERFORMANCE}

To evaluate the ability of the system to control a SPM we have used the system in conjunction with several different types of microscope available in our laboratory. Perhaps the instrument which best illustrates the capability of the control system is the ultrahigh vacuum scanning tunneling microscope (UHVSTM). We have recently constructed a new scanner and sample translator assembly for the UHVSTM. The new scanner is capable of motions six times larger along the $Z$ axis ( $2 \mu \mathrm{m}$ ) and ten times larger in the $X$ and $Y$ dimensions $(3 \mu \mathrm{m})$ than our previous scanner. The new scanner was limited by the electronic noise of the control electronics which were previously used and could only achieve atomic resolution with the servo control voltage was limited to a $30-\mathrm{V}$ range. When using the control system described in this paper, atomic resolution was achieved with a full $380-\mathrm{V}$ control range; the imaging capability was limited in this case by the mechanical stability of the scanner assembly, rather than by the control electronics. Additionally, the modular design of the system and its standard communication interface allowed simple integration of control electronics for the sample translator.

The use of data from the UHVSTM to illustrate the system performance avoids some of the questions raised of STM in air, and tip sharpness can be evaluated by field ion microscopy in this microscope thus avoiding concerns common to SFM. In Fig. 10 we show an image of highly oriented pyrolytic graphite (HOPG) taken with the UHVSTM controlled by the digital control system. The 


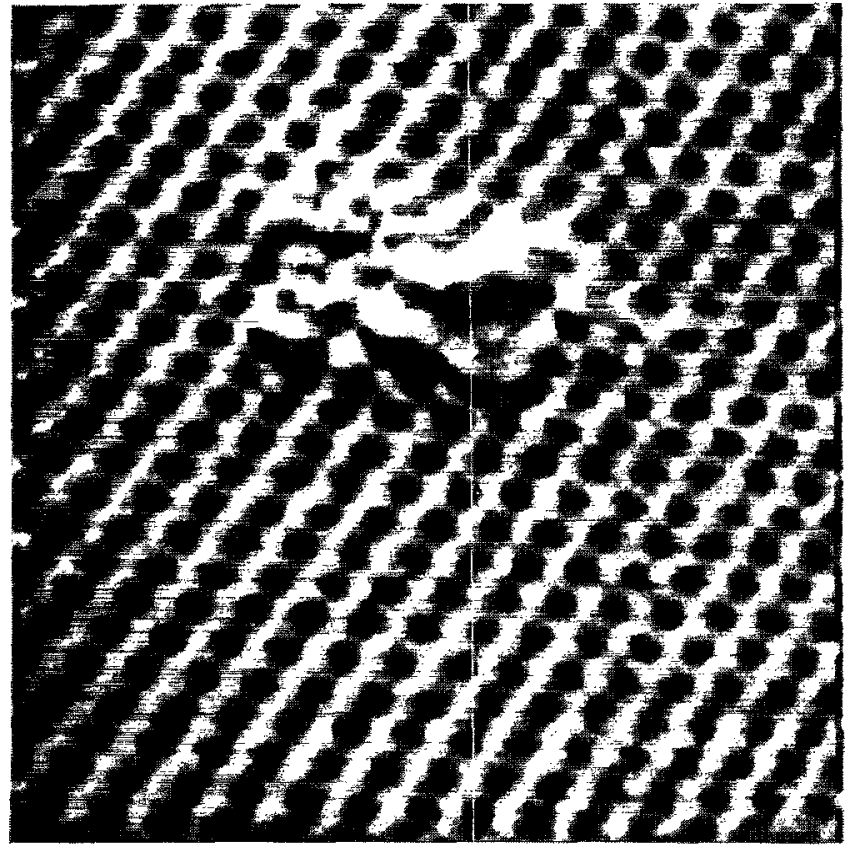

FIG. 10. An image of highly oriented pyrolytic graphite (HOPG) taken using the SPM control electronics presented in this article to control an ultrahigh vacuum (UHV) scanning tunneling microscope. A physisorbed contaminant is seen on the inert graphite basal plane. The image is approximately $50 \times 50 \AA$ with $250 \times 250$ pixeis acquired at 250 pixels $/ \mathrm{s}$. It is a constant current image (tunneling current $\approx 1 \mathrm{nA}$ ) at a sample bias of $-0.1 \mathrm{~V}$. The brightness in the image is proportional to both topographic height and slope. For details of image processing see the text.

data show atomic resolution as well as a physisorbed contaminant on the inert graphite basal plane. (During a subsequent scan, the contaminent was swept away midscan, thus affirming that it was weakly bound through physisorption rather than chemisorption.) The image is approximately $50 \times 50 \AA$ with $250 \times 250$ pixels acquired at 250 pixels/second. It is a constant current image acquired at a tunneling current of $1 \mathrm{nA}$ with a $-0.1-\mathrm{V}$ sample bias. The image is displayed with brightness proportional to both topographic height and slope. Tilt was removed by plane subtraction, and noise reduction was done through median filtering followed by smoothing with a binomially weighted sliding window 1-A square (full width at half maximum) which is small compared to the $2.4 \AA$ unit cell of HOPG.

\section{ADAPTATION OF THE SPM CONTROL AND DATA ACQUISITION SYSTEM TO NEAR FIELD SCANNING OPTICAL MICROSCOPY}

Finally, as an example of the flexibility afforded by modular design we consider the adaptation of the system to near field scanning optical microscopy (NSOM). NSOM holds great potential for imaging biomaterials because it provides all of the advantages of optical microscopy with subwavelength resolution. As a field of microscopy, NSOM is still evolving rapidly. With the need to implement quickly various imaging modes and feedback schemes, the flexibility provided by a modular SPM control system could be a tremendous advantage.
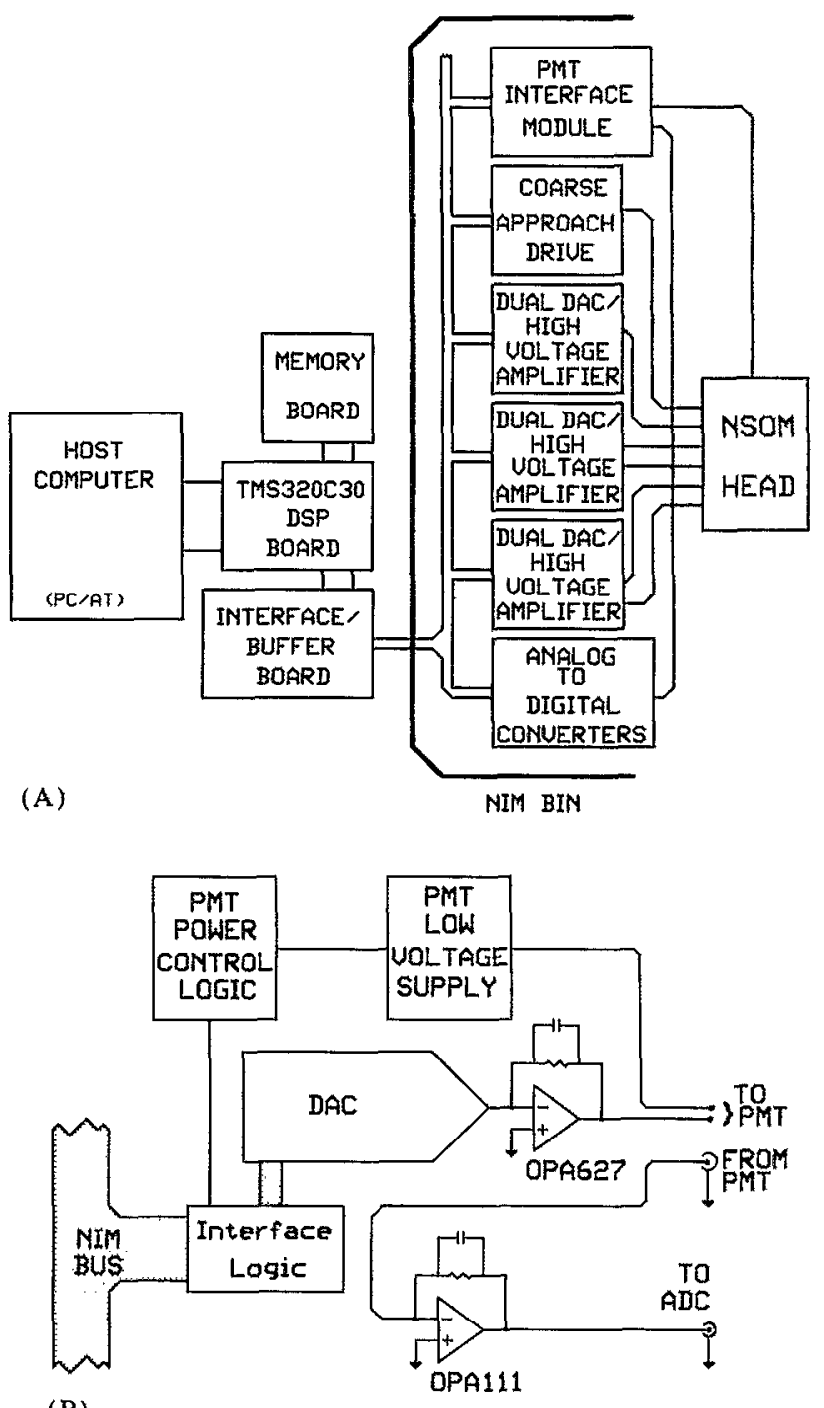

(B)

FIG. 11. A block diagram of the SPM control and data acquisition system as it might be adapted for ncar field scanning optical microscopy. The major addition needed to perform NSOM experiments is the photomultiplier tube (PMT) control and data acquisition module. (A) A diagram showing how the PMT module interfaces with the rest of the SPM system. (B) A more detailed diagram of the PMT module itself showing conceptually how such a module might be implemented.

The adaptation of the SPM control and data acquisition system to do NSOM is quite simple. In terms of the software, only minor reconfiguration is required. As for the hardware, the only additional requirement is that the instrument be able to detect very weak intensity light signals; thus there is a need for the ability to control and acquire data from photomultiplier tubes (PMTs). In Figs. 11(a) and 11(b) we present a diagram of how the control of and data acquisition from a PMT might be done. Figure 11(a) shows a diagram of how a PMT control module could be added to the system. In Fig. 11(b) we show a more detailed diagram of the contents of the PMT control module.

The relevant parameters for PMTS are the magnitude of the high voltage applied to the tube during use and the photocurrent that is generated. Currently, at least one manufacturer ${ }^{24}$ supplies a hybrid photodetector which con- 
sists of a PMT, a PMT socket, and a miniaturized switching mode power supply to provide the high voltage. The high voltage applied to the PMT may be varied by adjusting a low voltage control line via a DAC and the supply may be turned on and off by turning off the low voltage power to the detector. We have found these self-contained units to have excellent performance as well as being quite compact and convenient.

When photon counting is not required, acquisition of data from the PMT is easily done by using the standard current to voltage conversion circuit with a high input impedance amplifier. When using this type of circuit it is important to note that electrometer amplifiers tend to have significant output impedances and may require some buffering before the ADC input.

The adaptation of the SPM control system for NSOM is as simple as the construction and installation of a module to interface with PMTs. With this type of module both integrated light intensity and light modulation detection could easily be done.

\section{ACKNOWLEDGMENTS}

The authors would like to thank Jean-Paul Revel for a critical reading of the manuscript. Financial support for this work was provided by Abbott Laboratories, Inc., the National Institutes of Health Training Grant GM07617 (S.M.C.), the Ford Motor Company, and a National Science Foundation Fellowship (D.R.B.). The authors will gladly supply more detailed documentation to interested readers.
${ }^{1}$ J. H. Hoh, R. Lal, S. A. John, J..P. Revel, and M. F. Arnsdorf, Science 253, 1405 (1991).

${ }^{2}$ H. G. Hansma et al. Science (in press).

${ }^{3}$ A. Engel, Annu. Rev. Biophys. Biophys. Chem. 20, 79 (1991).

${ }^{4}$ C. Bustamante, J. Vesenka, C. Tang, W. Rees, M. Guthold, and R. Keller, Biochemistry 31, 22 (1992).

${ }^{5} \mathrm{~A}$. Lewis and K. Lieberman, Anal. Chem. 63, 625 A (1991) and references therein.

${ }^{6} \mathrm{C}$. Prater, P. Hansma, M. Tortonese, and C. Quate, Rev. Sci. Instrum. 62, 2634 (1991).

${ }^{7}$ A. Bard, F. Fan, D. Pierce, P. Unwin, D. Wipf, and F. Zhou, Science 254, 68 (1991).

${ }^{8}$ O. Marti, S. Gould, and P. K. Hansma, Rev. Sci. Instrum. 59, 836 (1988).

${ }^{9}$ A. Brown and R. W. Cline, Rev. Sci. Instrum. 61, 1484 (1990).

${ }^{10}$ R. S. Robinson, T. H. Kimsey, and R. Kimsey, J. Vac. Sci. Technol. B 9, 631 (1991).

${ }^{11}$ A. J. Hoeven, E. J. van Loenen, P. J. G. M. van Ilooft, and K. Oostveen, Rev. Sci. Instrum. 61, 1668 (1990).

${ }^{12}$ R. Piner and R. Reifenberger, Rev. Sci. Instrum. 60, 3123 (1989).

${ }^{13}$ R. C. Barrett and C. F. Quate, Rev. Sci. Instrum. 62, 1393 (1991).

${ }^{14}$ Motorola, Inc., P. O. Box 1466, Austin, TX 78767 USA.

${ }^{15}$ A. Ambrozy, Electronic Noise (McGraw-Hill, New York, 1982).

${ }^{16}$ Spectrum Signal Processing, Inc., \#301 3700 Gilmore Way, Burnaby, B.C. V5G4M1, Canada.

${ }^{17}$ D. Baselt, S. Clark, M. Youngquist, C. Spence, and J. Baldeschwieler, in preparation.

${ }^{18}$ Texas Instruments, Inc., P.O. Box 655012, Dallas, TX 75265 USA.

${ }^{19}$ Burr-Brown Corp., $6730 \mathrm{~S}$. Tucson Blvd., Tucson, AZ 85734 USA.

${ }^{20}$ Apex Microtechnology, Corp., 5980 N. Shannon Rd., Tucson, AZ 85741 USA.

${ }^{21}$ D. Sheingold, ed., Analog-Digital Handbook (Prentice-Hall, Englewood Cliffs, NJ, 1986).

${ }^{22}$ Toko America, Inc., 1250 Feehanville Dr., Mount Prospect, IL 60056 USA.

${ }^{23}$ Analog Devices, Inc., One Technology Way, P.O. Box 9106, Norwood, MA 02062 USA.

${ }^{24}$ Hamamatsu Photonics Corp., 360 Foothill Road, P.O. Box 6910, Bridgewater, N.J. 08807 USA.

${ }^{25}$ G. Franklin and J. Powell, Digital Control of Dynamic Systems (Addison-Wesley, Menlo Park, CA 1980). 